\title{
Atividade ovicida e larvicida in vitro do óleo essencial de Eucalyptus globulus sobre Haemonchus contortus
}

\author{
Ovicidal and larvicidal activity in vitro of Eucalyptus globulus essential oils on Haemonchus contortus
}

Iara T. F. Macedo ${ }^{1}$; Claudia M. L. Bevilaqua ${ }^{1 *}$; Lorena M. B. de Oliveira ${ }^{1}$;

Ana L. F. Camurça-Vasconcelos ${ }^{1}$; Luiz da S. Vieira²; Fabrício R. Oliveira ${ }^{1}$; Eudson M. Queiroz-Junior ${ }^{1}$; Bruno G. Portela ${ }^{1}$; Renata S. Barros ${ }^{1}$; Ana C. S. Chagas ${ }^{3}$

${ }^{1}$ Programa de Pós-Graduação em Ciências Veterinárias, Universidade Estadual do Ceará - UECE

${ }^{2}$ Empresa Brasileira de Pesquisa Agropecuária, Embrapa Caprinos e Ovinos - EMBRAPA/CNPC

${ }^{3}$ Empresa Brasileira de Pesquisa Agropecuária, Embrapa Pecuária Sudeste - EMBRAPA/CPPSE

Recebido em 27 de Janeiro de 2009

Aceito em 23 de Março de 2009

\section{Resumo}

O objetivo deste trabalho foi avaliar o efeito do óleo essencial de Eucalyptus globulus (OEEG) sobre a eclosão e desenvolvimento de larvas de Haemonchus contortus. A determinação da composição química do OEEG foi feita por meio de cromatografia gasosa acoplada à espectrometria de massa. No teste de eclosão de ovos (TEO), utilizaram-se as concentraçôes 21,$75 ; 17,4 ; 8,7 ; 5,43$ e $2,71 \mathrm{mg} \cdot \mathrm{mL}^{-1}$; e no de desenvolvimento larvar (TDL) as concentraçóes foram 43,$5 ; 21,75 ; 10,87 ; 5,43$ e $2,71 \mathrm{mg} \cdot \mathrm{mL}^{-1}$. Cada ensaio foi acompanhado por um controle positivo, $0,02 \mathrm{mg} \cdot \mathrm{mL}^{-1}$ tiabendazol, no TEO e $0,008 \mathrm{mg} \cdot \mathrm{mL}^{-1}$ ivermectina no TDL, e por um controle negativo com o diluente Tween 80 (3\%). A eficácia máxima obtida pelo OEEG sobre ovos foi de $99,3 \%$ na concentração de $21,75 \mathrm{mg} \cdot \mathrm{mL}^{-1}$ e, sobre larvas, $98,7 \%$ na concentração de $43,5 \mathrm{mg} \cdot \mathrm{mL}^{-1}$. A concentração do OEEG, que inviabilizou $50 \%$ dos ovos e das larvas do parasito, foi de 8,3 e $6,92 \mathrm{mg} \cdot \mathrm{mL}^{-1}$, respectivamente. A análise química do óleo identificou como principal componente o monoterpeno 1,8-cineol. O OEEG apresentou atividade ovicida e larvicida in vitro sobre $H$. contortus, determinando um bom potencial para utilização no controle de nematóides gastrintestinais de ovinos e caprinos.

Palavras-chave: Haemonchus contortus, fitoterapia, anti-helmíntico.

\begin{abstract}
The objective of this work was to evaluate ovicidal and larvicidal effects of Eucalyptus globulus essential oil (EGEO) on Haemonchus contortus. The chemical composition determination of EGEO was through gas chromatography and mass spectrometry. Egg hatch test (EHT) was performed in concentrations $21.75 ; 17.4 ; 8.7 ; 5.43$ e $2.71 \mathrm{mg} \cdot \mathrm{mL}^{-1}$. In larval development test (LDT) were used the concentrations 43.5; 21.75; 10.87; 5.43 e $2.71 \mathrm{mg} \cdot \mathrm{mL}^{-1}$. Each trial was conducted by negative control with Tween $80(3 \%)$ and positive control, $0.02 \mathrm{mg} \cdot \mathrm{mL}^{-1}$ of thiabendazole in EHT and $0.008 \mathrm{mg} \cdot \mathrm{mL}^{-1}$ of ivermectin in LDT. The maximum effectiveness of EGEO on eggs was $99.3 \%$ in concentration of $21.75 \mathrm{mg} \cdot \mathrm{mL}^{-1}$ and on larvae was $98.7 \%$ in concentration $43.5 \mathrm{mg} \cdot \mathrm{mL}^{-1}$. The concentration of EGEO that inhibits $50 \%$ of the eggs and larvae was 8.3 and $6.92 \mathrm{mg} \cdot \mathrm{mL}^{-1}$, respectively. The oil chemical analysis identified as main component the monoterpen 1,8-cineol. EGEO presented ovicidal and larvicidal activities in vitro, revealing a good potential for use in the control of sheep and goat gastrointestinal nematodes.
\end{abstract}

Keywords: Haemonchus contortus, phytoterapic, anthelmintic.

\section{Introdução}

As endoparasitoses gastrintestinais constituem-se no principal entrave da ovinocultura, em todo o mundo, especialmente, nas regiōes tropicais, onde os prejuízos econômicos são mais acentuados

*Autor para correspondência: Claudia Maria Leal Bevilaqua Programa de Pós-graduação em Ciências Veterinárias, Faculdade de Veterinária, Universidade Estadual do Ceará - UECE

Av. Paranjana, 1.700, CEP 60.740-903 Fortaleza - CE, Brasil

e-mail: claudia.bevilaqua@pesquisador.cnpq.br
(VIEIRA, 2008). Dentre os parasitos gastrintestinais, destaca-se o nematóide hematófago Haemonchus contortus, por causa da sua alta prevalência e patogenicidade (AROSEMENA et al., 1999). O comprometimento da produçấo ocorre em decorrência da perda de apetite, diarréia, anemia e em casos severos, morte do animal (ATHANASIADOU; KYRIAZAKIS, 2004).

Para tentar controlar e reduzir as perdas causadas pelas infecçôes por helmintos, são utilizados anti-helmínticos sintéticos 
(EGUALE et al., 2007). Entretanto, o rápido desenvolvimento de populaçóes de nematóides resistentes, associado ao alto custo, risco de resíduos nos alimentos e de contaminação ambiental tornou necessária a busca por novas alternativas de controle (HERD, 1996; MELO et al., 2003). Opçôes como a utilização de plantas medicinais pode representar uma alternativa para o controle das parasitoses gastrintestinais e minimizar alguns desses problemas, apresentando a vantagem de serem sustentáveis e ambientalmente aceitas (COSTA et al., 2008).

Eucalyptus globulus pertence à família Myrtaceae, espécie nativa da Austrália, difundida mundialmente, sendo considerada eficaz no tratamento de inflamaçóes pulmonares e da excessiva mucosidade (ROCHA; SANTOS, 2007). O uso do óleo essencial obtido a partir das suas folhas apresenta importância farmacêutica, sendo propostas múltiplas aplicaçôes medicinais, incluindo atividade anti-inflamatória, analgésica (SILVA et al., 2003) e antioxidante (CRUZ et al., 2005). Estudos demonstraram atividade desse óleo sobre bactérias e fungos (NAVARRO et al., 1996; TAKAHASHI et al., 2004; SALARI et al., 2006; CARMELLI et al., 2008), sobre o carrapato Boophilus microplus (CHAGAS et al., 2002), bem como atividade inseticida sobre Musca domestica (HALIM; MORSY, 2005), Pediculus humanus capitis (YANG et al., 2004), além dos coleópteros Acanthoscelides obtectus (PAPACHRISTOS; STAMOPOULOS, 2004), Zabrotes subfasciatus e Callosobruchus maculatus (BRITO et al., 2006).

O objetivo deste trabalho foi avaliar a atividade in vitro do óleo essencial de E. globulus sobre a eclosão de ovos e desenvolvimento larvar de $H$. contortus.

\section{Material e Métodos}

\section{Obtenção do óleo essencial}

Foi utilizada uma preparação comercial do óleo essencial de E. globulus (OEEG), adquirido de Dierberguer Óleos Essenciais Ltda (Barra Bonita - São Paulo, lote 0611218). Para aumentar a solubilidade em meio aquoso, o óleo foi diluído em Tween 80 a 3\%.

\section{Obtenção de ovos e larvas de $H$. contortus}

Dois ovinos sem raça definida foram mantidos em gaiolas metabólicas e tratados inicialmente com três anti-helmínticos de diferentes princípios ativos ${ }^{1}$, em dias alternados, para eliminar todos os nematóides gastrintestinais. Após a constatação da eliminação da infecção, aproximadamente 21 dias, foram inoculadas 5.000 larvas infectantes (L3) de H. contortus e, posteriormente, foram realizados exames coprológicos para confirmar o estabelecimento da infecção experimental.

\section{Teste de eclosão de ovos (TEO)}

Foram coletadas $10 \mathrm{~g}$ de fezes diretamente da ampola retal de ovino portador de infecção monoespecífica por $H$. contortus. As fezes

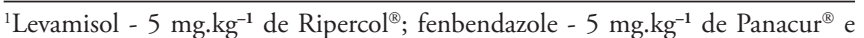
ivermectina - 0,2 mg. $\mathrm{kg}^{-1}$ de Ivomec ${ }^{\circledR}$
}

foram processadas de acordo com a técnica descrita por Hubert e Kerboeuf (1992) para recuperação de ovos. O teste de eclosão de ovos foi baseado na metodologia descrita por Coles et al. (1992). Aproximadamente, $250 \mu \mathrm{L}$ de solução de ovos, contendo no mínimo 100 ovos, foram incubados com $250 \mu \mathrm{L}$ da solução a ser testada de acordo com os seguintes tratamentos: T1: diluente Tween 80 a 3\%, controle negativo; T2: 0,02 mg. $\mathrm{mL}^{-1}$ tiabendazol, controle positivo; T3: óleo essencial de E. globulus nas concentraçôes 2,71; 5,43; 8,7; 17,4 e 21,75 mg.mL $\mathrm{mL}^{-1}$. Após 48 horas, foi acrescentado Lugol com o objetivo de interromper a eclosão das larvas e realizar a contagem de ovos e larvas. Foram realizadas três repetiçôes com cinco réplicas para cada tratamento.

\section{Teste de desenvolvimento larvar $(T D L)$}

Para a obtenção das larvas de primeiro estádio (L1), uma alíquota da suspensão de ovos obtida segundo Hubert e Kerboeuf (1992) foi incubada por 24 horas em estufa a $37^{\circ} \mathrm{C}$. O teste de desenvolvimento larvar foi realizado conforme método descrito por Roberts e O’Sullivan (1950) modificado. Uma alíquota de $1 \mathrm{~mL}$, contendo aproximadamente $250 \mathrm{~L} 1 \mathrm{de} H$. contortus, foi incubada durante 6 dias com $2 \mathrm{~g}$ de fezes provenientes de um animal livre de nematóides gastrintestinais, juntamente com $1 \mathrm{~mL}$ do óleo essencial de E. globulus nas concentraçóes 2,71; 5,43; 10,87; 21,75 e 43,5 mg.mL $\mathrm{mL}^{-1}$. O controle negativo foi constituído pelo diluente Tween 80 a 3\% e, o positivo, por ivermectina ${ }^{2}$. Ao final, foi acrescentado lugol e realizada a contagem de larvas de $3^{\circ}$ estádio. Foram realizadas três repetiçóes com cinco réplicas para cada tratamento.

\section{Análise química do óleo essencial}

A composição química do óleo essencial usado neste estudo foi determinada pela cromatografia gasosa acoplada à espectrometria de massa. O óleo essencial foi analisado, usando-se o equipamento Velocity XPT sob as seguintes condiçóes: coluna capilar de sílica fundida dimetilpolisiloxano DB $5 \mathrm{~ms}$; gás de arraste: $\mathrm{Ne}$ (1 $\mathrm{mL} / \mathrm{min}$ ); temperatura do injetor: $250{ }^{\circ} \mathrm{C}$; temperatura do detector: $200{ }^{\circ} \mathrm{C}$; temperatura da coluna: $35-180{ }^{\circ} \mathrm{C} \mathrm{a} 4{ }^{\circ} \mathrm{C} / \mathrm{min}$ e depois $180-25^{\circ} \mathrm{C}$ a $10{ }^{\circ} \mathrm{C} / \mathrm{min}$. Amostras foram analisadas pelo GCMS -QP2010, com impacto eletrônico a 70 eV.

\section{Análise estatística}

Os resultados foram expressos em percentagem de eficácia da inibição da eclosão de larvas e do seu desenvolvimento. Os resultados foram analisados usando ANOVA e comparados pelo teste de Tukey com nível de significância de 5\%, usando-se o programa Prism 3.0.

A eficácia de cada tratamento no teste de eclosão de ovos (E\%TEO) e do desenvolvimento larvar (E\%TDL) foi determinada segundo Camurça-Vasconcelos et al. (2007) de acordo com as Equaçóes 1 e 2. 


$$
\begin{aligned}
& \mathrm{E} \% \mathrm{TEO}=\frac{\text { número de larvas eclodidas }}{\text { número de larvas eclodidas }+ \text { número de ovos }} \times 100(1) \\
& \mathrm{E} \% \mathrm{TDL}=\frac{\mathrm{L} 3 \text { grupo controle negativo }-\mathrm{L} 3 \text { grupo tratado }}{\mathrm{L} 3 \text { grupo controle negativo }} \times 100(2)
\end{aligned}
$$

A concentração efetiva 50 (CE50), ou seja, a concentração capaz de inibir $50 \%$ da eclosão ou do desenvolvimento larvar foi calculada usando-se o programa estatístico SPSS 8.0 para Windows.

\section{Resultados}

As percentagens médias de eficácia do OEEG obtidas no teste de eclosão de ovos estão dispostas na Tabela 1. O OEEG na concentração de $17,4 \mathrm{mg} \cdot \mathrm{mL}^{-1}$ apresentou $87,3 \%$ de eficácia, não sendo diferente estatisticamente do anti-helmíntico ( $\mathrm{p}>0,05)$. A CE50 do OEEG foi de 8,3 (5,46-12,31) mg. $\mathrm{mL}^{-1}$.

A Tabela 2 apresenta as percentagens médias de eficácia do OEEG no teste de desenvolvimento larvar. $\mathrm{Na}$ concentração de $21,75 \mathrm{mg} \cdot \mathrm{mL}^{-1}$, o OEEG inibiu em $90,96 \%$ o desenvolvimento larvar, sendo equivalente à eficácia do anti-helmíntico $(\mathrm{p}>0,05)$. A CE50 do OEEG foi de 6,92 (5,12-9,01) mg. $\mathrm{mL}^{-1}$.

O óleo essencial apresentou efeito inibitório dose-dependente sobre a eclosão e desenvolvimento larvar de $H$. contortus.

A análise obtida por cromatografia gasosa indicou como principais constituintes e suas concentraçóes: $\alpha$-pineno $(4,15 \%)$, o-cimeno (2,93\%), (+)-limoneno (8,16\%), eucaliptol $(83,89 \%)$ e $\gamma$-terpineno $(0,87 \%)$.

\section{Discussão}

$\mathrm{O}$ modelo in vitro reportado neste estudo demonstrou o efeito anti-helmíntico do OEEG, sugerindo a presença de algum constituinte químico que atua sobre ovos e larvas de $H$. contortus. $\mathrm{O}$ uso de $H$. contortus, nesses testes, pode ser justificado pelo fato de que este nematóide é um parasito gastrintestinal de grande importância econômica na produção de pequenos ruminantes, apresentando alta prevalência e patogenicidade (DIEHL et al., 2004).

Os testes in vitro constituem-se na observação da ação do fármaco pelo contato direto com os estádios de ovo e/ou larva do parasito para avaliar seu efeito sobre a eclosão e desenvolvimento de larvas. A adição de uma substância aos ovos permite avaliar seu efeito sobre as mitoses tal como já descrito para os anti-helmínticos benzimidazóis (COLES et al., 1992), enquanto sobre as larvas causa morte por inanição devido à paralisia muscular, como já relatado para a ivermectina (GEARY et al., 1993), inibindo indiretamente o desenvolvimento das larvas. Atualmente, esses testes, além de verificar a ação de anti-helmínticos sintéticos, têm sido utilizados para triagem de plantas medicinais, apresentando as vantagens de facilidade de execução, baixo custo e rapidez. Esses ensaios servem como uma indicação inicial da atividade a ser pesquisada e permitem a seleção dos extratos mais promissores, diminuem gastos, evitam perda de tempo e o uso indiscriminado de animais de experimentação (CAMURÇA-VASCONCELOS et al., 2005).
Tabela 1. Percentual de eficácia média \pm erro-padrão (e.p.) do óleo essencial de Eucalyptus globulus na inibição da eclosão de larvas de Haemonchus contortus.

\begin{tabular}{cr}
\hline Concentraçóes $\left(\mathbf{m g} \cdot \mathbf{m L}^{-1}\right)$ & Eficácia \pm e.p \\
\hline 21,75 & $99,07 \pm 0,28 \mathrm{~A}$ \\
17,4 & $87,3 \pm 2,55 \mathrm{~B}$ \\
8,7 & $43,25 \pm 1,05 \mathrm{C}$ \\
5,43 & $21,87 \pm 2,27 \mathrm{D}$ \\
2,71 & $9,93 \pm 1,49 \mathrm{E}$ \\
Controle negativo & $3,12 \pm 0,45 \mathrm{E}$ \\
(3\% Tween 80) & $92,7 \pm 1,28 \mathrm{AB}$ \\
Controle positivo & \\
$\left(0,025\right.$ mg.mL ${ }^{-1}$ Tiabendazol $)$ &
\end{tabular}

Tabela 2. Percentual de eficácia média \pm erro-padrão (e.p.) do óleo essencial de Eucalyptus globulus na inibição do desenvolvimento larvar de Haemonchus contortus.

\begin{tabular}{cr}
\hline Concentraçóes $\left(\mathbf{m g} \cdot \mathbf{m L}^{-1}\right)$ & Eficácia \pm e.p \\
\hline 43,5 & $98,7 \pm 0,36 \mathrm{~A}$ \\
21,75 & $90,96 \pm 2,17 \mathrm{~A}$ \\
10,87 & $67,75 \pm 3,83 \mathrm{~B}$ \\
5,43 & $47,6 \pm 4,56 \mathrm{C}$ \\
2,71 & $8,37 \pm 2,08 \mathrm{D}$ \\
Controle negativo & $3,5 \pm 0,57 \mathrm{D}$ \\
$(3 \%$ Tween 80) & \\
Controle positivo & $99,81 \pm 0,1 \mathrm{~A}$ \\
\hline 0,008 mg.mL
\end{tabular}

Letras diferentes indicam significância estatística nas linhas $(\mathrm{p}<0,05)$.

Neste estudo, o OEEG apresentou atividade in vitro sobre H. contortus superior a outras plantas testadas. Como exemplo, é possível citar que $50 \mathrm{mg} \cdot \mathrm{mL}^{-1}$ do extrato hexânico das folhas de Melia azedarach inibiram em 16,92\% a eclosão de larvas e em $67,9 \%$ o desenvolvimento larvar, respectivamente (MACIEL et al., 2006); o extrato acetato de etila de Azadirachta indica inibiu em $51,31 \%$ a eclosão de larvas e em $68,10 \%$ o desenvolvimento larvar na concentração de 50 mg.mL $\mathrm{mL}^{-1}$ (COSTA et al., 2008); o extrato metanólico de Spigelia anthelmia inibiu em 97,4\% a eclosão de larvas e em $84,4 \%$ o desenvolvimento larvar na concentração de $50 \mathrm{mg} \cdot \mathrm{mL}^{-1}$ (ASSIS et al., 2003). Entretanto, o OEEG apresentou atividade inferior à de outras espécies do seu gênero, como o óleo essencial de E. staigeriana que inibiu a eclosão de larvas e o seu desenvolvimento nas concentraçóes de 10,6 e 2,65 mg. $\mathrm{mL}^{-1}$; e o óleo de E. citriodora, que apresentou concentraçôes efetivas de 5,4 e 2,7 mg. $\mathrm{mL}^{-1}$, sobre ovos e larvas, respectivamente (MACEDO, 2008).

Neste trabalho, o OEEG foi menos efetivo que os anti-helmínticos sintéticos usados como controle positivo. Entretanto, o tiabendazol e a ivermectina são substâncias ativas isoladas, enquanto o óleo essencial é uma mistura que contém vários constituintes químicos, dentre eles o princípio ativo com ação ovicida e larvicida. 
A investigação de componentes químicos provenientes de produtos naturais é de fundamental importância para o desenvolvimento de novas drogas anti-helmínticas (ASSIS et al., 2003). Neste estudo, a análise CG-SM identificou que o OEEG é composto por cinco constituintes químicos que podem ser responsáveis pela potencial atividade anti-helmíntica encontrada in vitro. $\mathrm{O}$ principal constituinte encontrado foi o monoterpeno 1,8-cineol, também conhecido por eucaliptol. Alguns trabalhos já foram realizados com esse composto para avaliar sua atividade inseticida sobre coleópteros Tribolium castanaeum (TRIPATHI et al., 2001; STAMOPOULOS et al., 2007), Sitophilus oryzae e Oryzaephilus surinamensis, sobre Musca domestica e Blattella germanica (LEE et al., 2003). Existem relatos sobre ação contra as bactérias Escherichia coli e Staphylococcus aureus (CARSON; RILLEY, 1995; CHA et al., 2007) sobre os fungos Candida albicans, Fusarium graminearum, F. culmorum e Pyrenophora graminea (TERZI et al., 2007) e larva do nematóide Anisakis simplex (NAVARRO et al., 2008).

O óleo essencial de E. globulus pode ser uma fonte alternativa para o controle de nematóides gastrintestinais. Entretanto, estudos são necessários para avaliar a sua toxicidade e seus efeitos in vivo, bem como isolamento e avaliação de seus componentes ativos.

\section{Referências}

AROSEMENA, N. A. E. et al. Seasonal variations of gastrointestinal nematodes in sheep and goats from semi-arid area in Brazil. Revue Médecine Vétérinaire, v. 150, n. 11, p. 873-876, 1999.

ASSIS, L. M. et al. Ovicidal and larvicidal activity in vitro of Spigelia anthelmia Linn. extracts on Haemonchus contortus. Veterinary Parasitology, v. 117, n. 1-2, p. 43-49, 2003.

ATHANASIADOU, S.; KYRIAZAKIS, I. Plant secondary metabolites: antiparasitic effects and their role in ruminant production systems. Proceedings of the Nutrition Society, v. 63, n. 4, p. 631-639, 2004.

BRITO, J. P.; OLIVEIRA, J. E. M.; DE-BORTOLI, S. A. Toxicidade de óleos essenciais de Eucalyptus spp. sobre Callosobruchus maculatus (Fabr., 1775) (Coleoptera: Bruchidae). Revista de Biologia e Ciências da Terra, v. 6, n. 1, p. 96-103, 2006.

CAMURÇA-VASCONCELOS, A. L. F. et al. Validação de plantas medicinais com atividade anti-helmíntica. Revista Brasileira de Plantas Medicinais, v. 7, n. 3, p. 97-106, 2005.

CAMURÇA-VASCONCELOS, A. L. F. et al. Anthelmintic activity of Croton zehntneri and Lippia sidoides essential oils. Veterinary Parasitology, v. 148, n. 3-4, p. 288-294, 2007.

CARMELLI, C. et al. Effect of Eucalyptus Essential Oil on Respiratory Bacteria and Viruses. Current microbiology, v. 56, n. 1, p. 89-92, 2008.

CARSON, C. F; RILLEY, T. V. Antimicrobial activity of the major components of the essential oiI of Melaleuca alternifolia. Journal of Applied Bacteriology, v. 78, n. 3, p. 264-269, 1995.

CHA, J. D. et al. Chemical Composition and Antibacterial Activity of Essential Oil from Artemisia feddei. Journal of Microbiology and Biotechnology, v. 17, n. 12, p. 2061-2065, 2007.

CHAGAS, A. C. S. et al. Efeito acaricida de óleos essenciais e concentrados emulsionáveis de Eucalyptus spp em Boophilus microplus.
Brazilian Journal of Veterinary Research and Animal Science, v. 39, n. 5, p. 247-253, 2002.

COLES, G. C. C. et al. World Association for the Advancement of Veterinary Parasitology (W.A.A.V.P.) methods for the detection of anthelmintic resistance in nematodes of veterinary importance. Veterinary Parasitology, v. 44, n. 1-2, p. 35-44, 1992.

COSTA, C. T. C. et al. In vitro ovicidal and larvicidal activity of Azadirachta indica extracts on Haemonchus contortus. Small Ruminant Research, v. 74, n. 1-3, p. 284-287, 2008

CRUZ, J. M.; DOMINGUEZ, H.; PARAJO, J. C. Anti-oxidant activity of isolates from acid hydrolysates of Eucalyptus globulus wood. Food Chemistry, v. 90, n. 4, p. 503-511, 2005.

DIEHL, M. S. et al. Prospect for anthelminthic plants in the Ivory Coast using ethnobotanical criteria. Journal of Ethnopharmacology, v. 95, n. 2-3, p. 277-284, 2004.

EGUALE, T. et al. In vitro and in vivo anthelmintic activity of crude extracts of Coriandrum sativum against Haemonchus contortus. Journal of Ethnopharmacology, v. 110, n. 3, p. 428-433, 2007.

GEARY, T. G. et al. Haemonchus contortus: Ivermectin-Induced Paralysis of the Pharynx. Experimental Parasitology, v. 77, n. 1, p. 88-96, 1993.

HERD, R. Impactos ambientais associados aos compostos endectocidas. In: Controle dos nematódeos gastrintestinais em ruminantes. PADILHA, T. (Ed.). Coronel Pacheco: EMBRAPA-CNPGL, 1996. p. $95-111$

HUBERT, J.; KERBOEUF, D. A microlarval development assay for the detection of anthelmintic resistance in sheep nematodes. Veterinary Record, v. 130, n. 20, p. 442-446, 1992.

LEE, S.; PETERSON, C. J.; COATS, J. R. Fumigation toxicity of monoterpenoids to several stored product insects. Journal of Stored Products Research, v. 39, n. 1, p. 77-85, 2003.

MACEDO, I. T. F. Atividade anti-helmíntica de óleos essenciais de Eucalyptus spp sobre nematóides gastrintestinais. Fortaleza, 2008. $87 \mathrm{f}$. Dissertação (Mestrado) - Universidade Estadual do Ceará. Disponível em:<http: www.uece.br/ppgcv/dmdocuments/iara_macedo.pdf $>$.

MACIEL, M. V. et al. Ovicidal and larvicidal activity of Melia azedarach extracts on Haemonchus contortus. Veterinary Parasitology, v. 140, n. 1-2, p. 98-104, 2006

MELO, A. C. F. L. et al. Nematóides resistentes a anti-helmíntico em rebanhos de ovinos e caprinos do estado do Ceará, Brasil. Ciência Rural, v. 37, n. 2, p. 339-344, 2003.

HALIM, A. S. A.; MORSY, T. A. The insecticidal activity of Eucalyptus globulus oil on the development of Musca domestica third stage larvae. Journal of the Egyptian Society of Parasitology, v. 35, n. 2, p. 631-636, 2005.

NAVARRO, V. et al. Antimicrobial evaluation of some plants used in Mexican traditional medicine for the treatment of infectious diseases. Journal of Etnopharmacology, v. 53, n. 3, p. 143-147, 1996.

NAVARRO, M. C. et al. Anisakis simplex s.l.: Larvicidal activity of various monoterpenic derivatives of natural origin against $\mathrm{L}_{3}$ larvae in vitro and in vivo. Experimental Parasitology, v. 120, n. 4, p. 295-299, 2008.

PAPACHRISTOS, D. P.; STAMOPOULOS, D.C. Fumigant toxicity of three essential oils on the eggs of Acanthoscelides obtectus (Say) 
(Coleoptera: Bruchidae). Journal of Stored Products Research, v. 40, n. 5 , p. 517-525, 2004.

ROBERTS, F. H. S.; O'SULLIVAN, P. J. Methods for egg counts and larval cultures for strongyles infecting the gastrointestinal tract of cattle. Australian Journal of Agricultural Research, v. 1, n. 1, p. 99-102, 1950.

ROCHA, M. E. N.; SANTOS, C. L. O uso commercial e popular do eucalipto Eucalyptus globulus Labill- Myrtaceae. Saúde \& Ambiente em Revista, v. 2, n. 2, p. 23-34, 2007.

SALARI, M. H. et al. Antibacterial effects of Eucalyptus globulus leaf extract on pathogenic bacteria isolated from specimens of patients with respiratory tract disorders. Clinical Microbiology and Infection, v. 12, n. 2, p. 194-196, 2006.

SILVA, J. et al. Analgesic and anti-inflammatory effects of essential oils of Eucalyptus. Journal of Ethnopharmacology, v. 89, n. 2-3, p. 277-283, 2003.

STAMOPOULOS, D. C.; DAMOS, P.; KARAGIANIDOU, G. Bioactivity of five monoterpenoid vapours to Tribolium confusum (du Val) (Coleoptera: Tenebrionidae). Journal of Stored Products Research, v. 43 , n. 4 , p. 571-577, 2007.
TAKAHASHI, T.; KOKUBO, R.; SAKAINO, M. Antimicrobial activities of eucalyptus leaf extracts and flavonoids from Eucalyptus maculata. Letters in Applied Microbiology, v. 39, n. 1, p. 60-64, 2004 .

TERZI, V. et al. In vitro antifungal activity of the tea tree (Melaleuca alternifolia) essential oil and its major components against plant pathogens. Letters in Applied Microbiology, v. 44, n. 6, p. 613-618, 2007.

TRIPATHI, A. K. et al. Toxicity, Feeding Deterrence, and Effect of Activity of 1,8-Cineole from Artemisia annua on Progeny Production of Tribolium castanaeum (Coleoptera: Tenebrionidae). Journal of Economic Entomology, v. 94, n. 4, p. 979-983, 2001.

VIEIRA, L. S. Métodos alternativos de controle de nematóides gastrintestinais em caprinos e ovinos. Tecnologia \& Ciências Agropecuárias, v. 2, n. 2, p. 49-56, 2008.

YANG, Y. C. et al. Ovicidal and Adulticidal Activity of Eucalyptus globulus Leaf Oil Terpenoids against Pediculus humanus capitis (Anoplura: Pediculidae). Journal of Agricultural and Food Chemistry, v. 52, n. 9 , p. 2507-2511, 2004. 